\title{
The potential differential diagnosis value and clinical significance of CD35 expression in B-chronic lymphoproliferative disorders
}

\author{
Yu Shi", Lu Liu", Hui Yang", Xiao Chen, Yan Wang, Sishu Zhao, Huimin Jin, Yujie Wu \\ Department of Hematology, The First Affiliated Hospital of Nanjing Medical University (Jiangsu Provincial People's Hospital), Nanjing, China \\ Contributions: (I) Conception and design: Y Wu; (II) Administrative support: None; (III) Provision of study materials or patients: None; (IV) \\ Collection and assembly of data: All authors; (V) Data analysis and interpretation: All authors; (VI) Manuscript writing: All authors; (VII) Final \\ approval of manuscript: All authors. \\ "These authors contributed equally to this work. \\ Correspondence to: Yujie Wu. Department of Hematology, The First Affiliated Hospital of Nanjing Medical University (Jiangsu Provincial People's \\ Hospital), Nanjing 210029, China. Email: wuyujie123456@163.com.
}

\begin{abstract}
Background: Flow cytometry for immunophenotyping is the main method for diagnosing chronic lymphocytic leukemia (CLL). Differential diagnosis between CLL and other B-chronic lymphoproliferative disorders (B-CLPDs) is sometimes difficult. This study aimed to investigate whether cluster of differentiation 35 (CD35) could be a useful marker for the differential diagnosis of CLL and other B-CLPDs.

Methods: The CD35 expression on lymphoma cells from 516 B-CLPD patients (347 CLL, 169 other B-CLPDs) was investigated through flow cytometry analysis. Serum C3 and C4 levels in B-CLPD patients were also evaluated.
\end{abstract}

Results: The results showed that the expression percentage and mean fluorescence intensity of CD35 were reduced in CLL cases compared with other B-CLPD patients. Furthermore, CD35 < $17 \%$ produced a sensitivity of $81.8 \%$ and a specificity of $88.4 \%$ for supporting the diagnosis of CLL. Additionally, the addition of CD35 to Matutes score improved the score's discriminative power. The sensitivity of the Matutes score was improved from $81.3 \%$ to $88.5 \%$, and the accuracy was improved from $96.6 \%$ to $97.6 \%$. Finally, $15.0 \%$ and $16.4 \%$ of CLL patients had defective serum C3 and C4 levels at diagnosis, respectively.

Conclusions: Evaluating CD35 expression could have potential differential diagnostic value in distinguishing CLL from other B-CLPDs, especially between CLL and mantle cell lymphoma (MCL).

Keywords: CD35; flow cytometry; chronic lymphocytic leukemia (CLL); differential diagnosis; B-chronic lymphoproliferative disorders (B-CLPDs)

Submitted May 25, 2021. Accepted for publication Jul 14, 2021.

doi: 10.21037/atm-21-3199

View this article at: https://dx.doi.org/10.21037/atm-21-3199

\section{Introduction}

Chronic lymphocytic leukemia (CLL) is a subtype of B-chronic lymphoproliferative disorders (B-CLPDs) and has specific immunophenotypic properties. It mainly occurs in older adults and is characterized by the accumulation of malignant cluster of differentiation 5 -positive $\left(\mathrm{CD}^{+}\right)$ B lymphocytes in the blood, bone marrow, spleen, and lymphoid organs (1-3). The definitive diagnosis of CLL is mainly based on the following 3 criteria: (I) peripheral blood monoclonal B lymphocyte count reaching $\geq 5 \times 10^{9} / \mathrm{L}$; (II) morphologic features of a marked increase in small and mature lymphocytes from peripheral smear; and a (III) typical immunophenotype of $\mathrm{CD} 19^{+}, \mathrm{CD}^{+}, \mathrm{CD} 23^{+}$, $\mathrm{CD}^{200^{+}}$, weak CD22, CD79b (dim), $\mathrm{FMC7}^{-}$, and weak surface immunoglobulin (sIg) expression (dim) (4). Multiparameter flow cytometry for immunophenotyping is the main method for the differential diagnosis between CLL and other B-CLPDs. The flow cytometry score, developed by Estella Matutes, is widely used, and CLL typically 
displays a score of 4 or 5 (5-7). However, some CLL cases may have an atypical immunophenotype with a Matutes score of less than 4, which usually results in diagnostic difficulties. In the leukemic phase, CLL and other B-CLPDs, especially $\mathrm{CD}^{+} \mathrm{B}$-cell neoplasms, which are mainly mantle cell lymphoma (MCL), can share some similar features. However, their clinical and prognostic features are significantly different (8-11). Therefore, the addition of novel immunophenotypic marker(s) that can help identify B-CLPDs may have considerable clinical value.

CD35, also known as complement receptor 1 (CR1), is a cell surface glycoprotein distributed on the surface of a variety of cells, including red blood cells, granulocytes, monocytes, mast cells, follicular dendritic cells, glomerular foot process cells, B cells and some CD4+ $\mathrm{T}$ cells. The high- and low-affinity ligands of CD35 are C3b/C4b and $\mathrm{C} 3 \mathrm{bi} / \mathrm{C} 3 \mathrm{c}$, respectively (12-14). It has been reported that $\mathrm{CD} 35$ is also a receptor for $\mathrm{C} 1 \mathrm{q}$, but the role of CD35 as a C1q receptor remains to be further studied (15). CD35 has an immune adhesion function, and can mediate the combination of immune complexes and red blood cells, monocytes, neutrophils, macrophages, B lymphocytes, or eosinophils in a covalent form. CD35 can also accelerate the degradation of the $\mathrm{C} 3$ and $\mathrm{C} 5$ convertases, further regulating complement system activation. In addition, CD35 serves as a cofactor, participating in the limited cleavage of $\mathrm{C} 4 \mathrm{~b}$ and $\mathrm{C} 3 \mathrm{~b}$, with the resulting fragments being the ligands of other complement receptors $(16,17)$. A previous study reported that the expression of CD35 on B CLL cells was reduced or even negative in patients with CLL (18). This suggests that investigations into the addition of CD35 expression in the differential diagnosis of B-CLPDs could help to reach a definitive conclusion which can distinguish CLL from other B-CLPDs.

In this study, the significance of CD35 expression in leukemic B cells of patients with CLL and other B-CLPDs was investigated. The results confirmed that the surface expression of CD35 on leukemic B cells was reduced or even negative in patients with CLL compared with other B-CLPDs. The cut-off value of CD35 in the differential diagnosis of CLL was also determined and the addition of CD35 to Matutes score improved the score's discriminative power. Consequently, CD35 has been comprehensively proved to be a useful marker for the differential diagnosis between CLL and other mature B-cell neoplasms, especially between CLL and MCL. We present the following article in accordance with the STARD reporting checklist (available at https://dx.doi.org/10.21037/atm-21-3199).

\section{Methods}

\section{B-CLPD characteristics}

In the research, peripheral blood (PB) or bone marrow (BM) samples collected from patients diagnosed with B-CLPDs from January 2015 to February 2020 were examined. According to the World Health Organization (WHO) 2016 classification of Tumors of Haematopoietic and Lymphoid Tissues and its revisions, consecutive B-CLPD patients were further diagnosed as CLL, MCL, follicular lymphoma (FL), waldenstrom macroglobulinemia (WM), marginal zone lymphoma (MZL), or hairy cell leukemia (HCL) (19). The study was conducted in accordance with the Declaration of Helsinki (as revised in 2013). The study was approved by the institutional ethics board of Jiangsu Province Hospital (No. 2021-SRFA-208) and individual consent for this retrospective analysis was waived.

B-CLPDs are classified according to peripheral blood cell count, morphological analysis of peripheral blood or bone marrow, and immunophenotyping of cells by flow cytometry. CLL is diagnosed by the standard criteria of microscopic cell morphology and flow cytometry analysis with calculation of the Matutes score. For typical CLL cases, the Matutes score is 4 or 5 , while atypical CLL cases are identified by a score $<4$. The expression of cyclin $\mathrm{D} 1$, the translocation of $I G H / C C N D 1$ or the presence of $\mathrm{t}(11 ; 14)$ is used to either diagnose or exclude MCL, while the presence of $t(14 ; 18)$ is used to diagnose FL. "Hair cells" can be seen in the peripheral blood, bone marrow, liver, or spleen of patients with HCL. The diagnosis of MZL requires histopathological examination of the corresponding tissues.

\section{Immunophenotypic analysis}

Fresh $\mathrm{PB}$ and $\mathrm{BM}$ samples were examined by flow cytometric analysis according to the procedure described below. First, $2 \mathrm{~mL}$ of $\mathrm{PB}$ or BM was aseptically drawn into anticoagulant tubes containing ethylene diamine tetraacetic acid (EDTA). Then, $100 \mu \mathrm{L}$ of $\mathrm{PB} / \mathrm{BM}$ was taken and washed with phosphate-buffered saline (PBS) to remove the free light chains, after which $500 \mu \mathrm{L}$ of erythrocyte lysate was added. After 10 minutes, the same amount of PBS was added and mixed. The supernatant was removed 5 minutes later, and then $1 \mathrm{~mL}$ of PBS was added for washing. Next, the appropriate monoclonal antibodies (mAbs) were added. After being incubated for 15 minutes at room temperature in darkness, the samples were washed with PBS and 
resuspended with $500 \mu \mathrm{L}$ of PBS. At least 50,000 cells were analyzed by flow cytometer (Beckman Coulter, USA).

The mAbs applied in the study were CD45, CD19, CD5, CD23, CD10, CD22, CD20, CD79b, FMC7, CD103, CD11c, CD25, CD38, CD49d, CD138, CD200 (Immunotech SA, Marseilles, France), and SmIg (Dako, Glostrup, Denmark). Corresponding isotype antibodies were used as a negative control. In addition, CD35 (BD Biosciences, NJ, USA) was tested in all cases.

The lymphocyte population was defined as low forward scatter/low side scatter/CD $45^{\text {high }}$, and subsequent analysis was conducted on $\mathrm{CD} 19^{+}$B cells. Positivity was identified as a $\geq 30 \%$ positive cell population. In general, sIgM and CD22 were counted as a score of 1 when its expression was weak. CD5 and CD23 were counted as a score of 1 when the positive cell population was $\geq 30 \%$. FMC7 was counted as a score of 1 when the positive cell population was $<30 \%$. In addition, the weak, moderate, or high expression pattern was assessed based on the $\log$ scale of the fluorescence axis on the B-cell population. The gating strategy involved specifically identifying CD35 on CD19 single-positive or $\mathrm{CD}^{+} \mathrm{CD} 19^{+}$double-positive lymphoma cells, depending on the subtype of B-CLPDs. The CD35 expression percentage and mean fluorescence intensity (MFI) were calculated in lymphoma cells by analysis software after the flow cytometry data were acquired.

\section{Measurement of complement $C 3$ and C4}

The blood samples were collected within 24 hours for measuring complement $\mathrm{C} 3$ and $\mathrm{C} 4$. The concentration of complement $\mathrm{C} 3$ and $\mathrm{C} 4$ was detected by immune scatter turbidimetry (normal values: C3 $0.79-1.52 \mathrm{~g} / \mathrm{L}$; C4 0.16 $0.38 \mathrm{~g} / \mathrm{L})$. Measurements were conducted using the BN2 special protein analyzer and matching reagents (Siemens AG, Munich, Germany), according to the manufacturer's protocol.

\section{Statistical analysis}

All statistical analyses were performed by Graphpad Prism 8 (GraphPad Software, San Diego, CA, USA) and SPSS version 22.0 (IBM Corp., Armonk, NY, USA). The comparison of CD35 expression among B-CLPD groups was calculated by the Kruskal-Wallis test. Statistical significance was defined as a $\mathrm{P}$ value less than 0.05 . A receiver operating characteristic curve (ROC) and area under the ROC curve (AUC) were established to evaluate the diagnostic value of
CD35 in differentiating the B-CLPD subtypes.

\section{Results \\ Biologic and laboratory characteristics}

A total of 516 patients diagnosed with B-CLPDs were included in the study. The most common subtype was CLL $(67.2 \%, n=347)$, followed by MCL (13.4\%, $n=69)$, WM $(7.2 \%, n=37)$, FL (5.8\%, $n=30)$, MZL $(4.5 \%, n=23)$, and HCL $(1.9 \%, \mathrm{n}=10)$. The median age was 62 years old (range, 27-86) at diagnosis. The male:female ratio of B-CLPDs patients was 2.2:1. The male patients were prevalent in the CLL, MCL, MZL and WM subgroups, but there was little difference between males and females in FL and HCL subgroups (Table 1).

Anemia and thrombocytopenia were common in B-CLPD patients. Anemia [hemoglobin (HGB) $<120 \mathrm{~g} / \mathrm{L}$ in males; HGB $<110 \mathrm{~g} / \mathrm{L}$ in females] occurred in $38.9 \%$ of patients while thrombocytopenia [platelet (PLT) $\left.<100 \times 10^{9} / \mathrm{L}\right]$ was observed in $30.0 \%$ of patients. Anemia occurred in $89.2 \%$ of patients with WM, which was the most common subtype. Severe anemia (HGB $<60 \mathrm{~g} / \mathrm{L})$ was also more likely to occur in WM patients, but rarely occurred in patients with MZL, FL, and CLL (less than 2.6\%). Thrombocytopenia most often occurred in WM patients, with an incidence rate of $32.4 \%$. However, only a slight proportion of patients with thrombocytopenia suffered from the severe deficiency associated with the condition. Hyperleukocytosis [white blood cell (WBC) $\left.>10 \times 10^{9} / \mathrm{L}\right]$ was observed in some patients with CLL $\left(11.0 \%\right.$ ), while leukopenia (WBC $<4 \times 10^{9} / \mathrm{L}$ ) occurred in $45.9 \%$ and $50 \%$ of WM and HCL patients, respectively. The non-hematological laboratory characteristics, including B symptoms, hepatomegaly, splenomegaly, and lymphadenopathy at diagnosis, are also listed in Table 1. Approximately $35.3 \%$ of patients had B symptoms. Splenomegaly was commonly apparent in patients with MCL (44.9\%) and MZL (39.1\%). The incidence of hepatomegaly was not high in any of the B-CLPD subtypes. Lymphadenopathy was more common in patients with CLL (56.5\%), MCL (47.8\%), and FL (66.7\%).

\section{The expression pattern of CD35 in B-CLPDs}

CD35 expression was tested in 516 B-CLPD cases. The $\mathrm{CD} 5^{+} \mathrm{CD} 19^{+}$double-positive lymphoma cells showed weak CD35 expression in CLL patients (Figure 1). The 
Table 1 The clinical characteristics of patients with B-CLPDs $(\mathrm{n}=516)$

\begin{tabular}{|c|c|c|c|c|c|c|}
\hline Characteristic & CLL & MCL & $\mathrm{FL}$ & MZL & WM & $\mathrm{HCL}$ \\
\hline Male/female & $2.0 / 1$ & $3.3 / 1$ & $0.9 / 1$ & $4.8 / 1$ & $3.6 / 1$ & $1.5 / 1$ \\
\hline WBC $\left(\times 10^{9} / \mathrm{L}\right)$, mean [range] & $46.2[0.7-540.4]$ & $32.3[1.3-518.0]$ & $14.3[2.6-80.4]$ & $49.4[2.8-746.1]$ & $4.5[0.8-9.9]$ & $12.9[1.6-59.3]$ \\
\hline ALC $\left(\times 10^{9} / L\right)$, mean [range] & $39.8[0.3-470.4]$ & $25.8[0.6-431]$ & $8.9[0.1-74.3]$ & $42.2[0.9-696.1]$ & $1.5[0.3-5.5]$ & $10.1[0.7-52.8]$ \\
\hline PLT $\left(\times 10^{9} / \mathrm{L}\right)$, mean [range] & $133.2[2-451]$ & $137.6[14-411]$ & $174.5[78-418]$ & $155.9[15-426]$ & 153. 9 [15-312] & $132.2[42-259]$ \\
\hline B symptom, n (\%) & $115(33.1)$ & $34(49.3)$ & $8(26.7)$ & $11(47.8)$ & $12(32.4)$ & $2(20.0)$ \\
\hline Hepatomegaly, n (\%) & $14(4.0)$ & $6(8.7)$ & $3(10.0)$ & $1(4.3)$ & $2(5.4)$ & $1(10.0)$ \\
\hline Splenomegaly, n (\%) & $90(25.9)$ & $31(44.9)$ & $4(13.3)$ & $9(39.1)$ & 7 (18.9) & $2(20.0)$ \\
\hline
\end{tabular}

B-CLPD, B-chronic lymphoproliferative disorder; CLL, chronic lymphocytic leukemia; MCL, mantle cell lymphoma; FL, follicular lymphoma; MZL, marginal zone lymphoma; WM, waldenstrom macroglobulinemia; HCL, hairy cell leukemia; WBC, white blood cell; ALC, absolute lymphocyte count; HGB, hemoglobin; PLT, platelet.
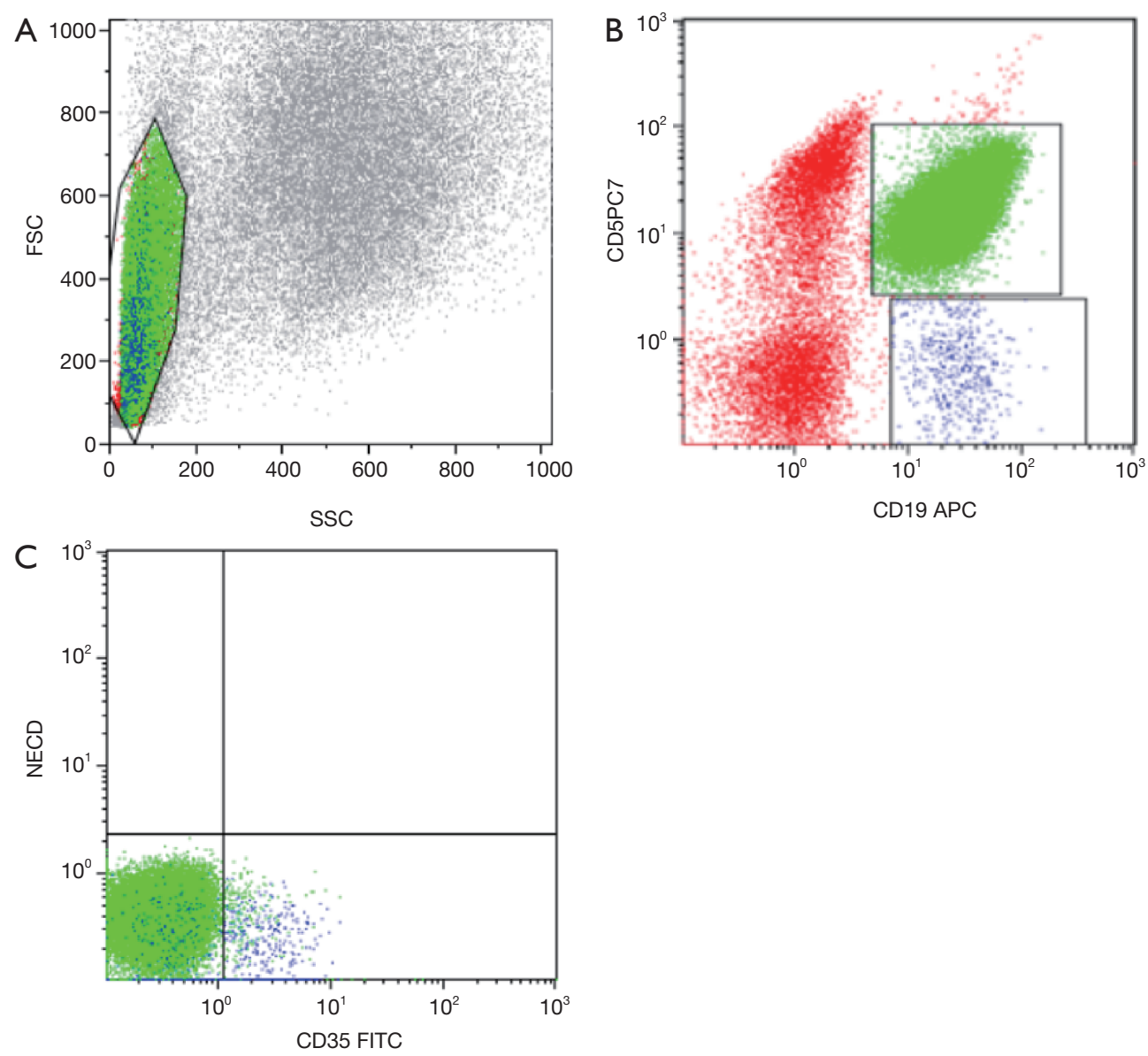

Figure 1 Flow cytometry measurements of B cells or lymphoma cells in a representative case of CLL using multiple antibodies. The low forward scatter/low side scatter/CD19+/CD5+ lymphoma cells of this patient with CLL show weak CD35 expression compared to low forward scatter/low side scatter/CD19+/CD5- B cells. CLL, chronic lymphocytic leukemia; CD, cluster of differentiation. 
A

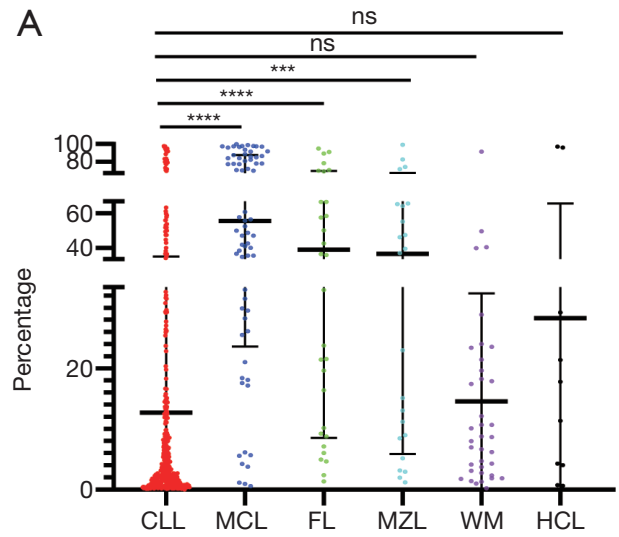

B

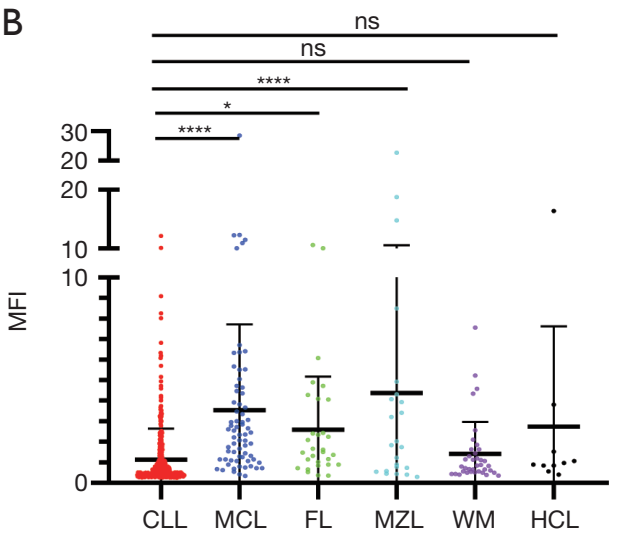

Figure 2 The distribution of CD35 expression in B-CLPDs. (A) The expression percentage of CD35 on leukemic B cells in B-CLPDs. (B) CD35 MFI of leukemic B cells for B-CLPDs. Error bars: mean \pm SEM. ${ }^{*} \mathrm{P}<0.05$, ${ }^{* * *} \mathrm{P}<0.001$, and ${ }^{* * * *} \mathrm{P}<0.0001$; ns: no significant difference between the two groups. CD, cluster of differentiation; B-CLPDs; B-chronic lymphoproliferative disorders; MFI, mean fluorescence intensity.

Table 2 The MFI and expression percentage of CD35 in B-CLPD patients

\begin{tabular}{|c|c|c|c|c|c|c|}
\hline Variable & CLL & MCL & $\mathrm{FL}$ & MZL & WM & $\mathrm{HCL}$ \\
\hline \multicolumn{7}{|l|}{ MFI } \\
\hline Mean & 1.13 & 3.54 & 2.59 & 4.37 & 1.41 & 2.73 \\
\hline Median & 0.54 & 2.36 & 1.56 & 1.83 & 0.83 & 0.93 \\
\hline \multicolumn{7}{|c|}{ Interquartile ranges } \\
\hline Min & 0.24 & 0.34 & 0.35 & 0.29 & 0.36 & 0.4 \\
\hline P25 & 0.38 & 1.14 & 0.92 & 0.66 & 0.55 & 0.84 \\
\hline P50 & 0.54 & 2.36 & 1.56 & 1.83 & 0.83 & 0.93 \\
\hline P75 & 1.08 & 4.36 & 3.66 & 4.19 & 1.45 & 1.41 \\
\hline Max & 12.1 & 28.49 & 10.6 & 22.68 & 7.57 & 16.4 \\
\hline \multicolumn{7}{|c|}{ Percentage (\%) } \\
\hline Mean & 12.7 & 55.5 & 38.8 & 36.5 & 14.6 & 28.2 \\
\hline Median & 2.89 & 55.5 & 34.31 & 36.92 & 8.66 & 14.56 \\
\hline \multicolumn{7}{|c|}{ Interquartile ranges } \\
\hline Min & 0.03 & 0.61 & 1.38 & 1.21 & 0.23 & 0.63 \\
\hline P25 & 0.98 & 29.85 & 9.46 & 8.74 & 3.11 & 4.12 \\
\hline P50 & 2.89 & 55.5 & 34.31 & 36.92 & 8.66 & 14.56 \\
\hline P75 & 11.51 & 84.89 & 66.40 & 64.64 & 19.64 & 27.24 \\
\hline Max & 97.58 & 99.77 & 94.93 & 99.16 & 91.35 & 96.95 \\
\hline
\end{tabular}

MFI, mean fluorescence intensity; B-CLPD, B-chronic lymphoproliferative disorder; CLL, chronic lymphocytic leukemia; MCL, mantle cell lymphoma; FL, follicular lymphoma; MZL, marginal zone lymphoma; WM, waldenstrom macroglobulinemia; $\mathrm{HCL}$, hairy cell leukemia. expression percentage and mean fluorescence intensities of CD35 were studied in B-CLPD patients. The distribution characteristics of CD35 in B-CLPD patients are shown in Figure 2. The mean MFI of CD35 in CLL, MCL, FL, MZL, WM, and HCL was 1.13 (range, 0.24-12.1), 3.54 (range, 0.34-28.49), 2.59 (range, 0.35-10.6), 4.37 (range, 0.29-22.68), 1.41 (range, 0.36-7.57), and 2.73 (range, 0.4-16.4), respectively. Meanwhile, the mean expression percentage of CD35 in CLL, MCL, FL, MZL, WM, and HCL was $12.7 \%$ (range, $0.03-97.58 \%$ ), $55.5 \%$ (range, 0.61-99.77\%), 38.8\% (range, 1.38-94.93\%), 36.5\% (range, 1.21-99.16\%), 14.6\% (range, 0.23-91.35\%), and $28.2 \%$ (range, $0.63-96.95 \%$ ) respectively. The median and interquartile ranges of CD35 MFI and expression percentage in CLL, MCL, FL, MZL, WM, and HCL are also shown in Table 2. CD35 MFI and expression percentage of CLL were lower than those of MCL, FL, and MZL, and the differences were statistically significant. Although the CD35 MFI and expression percentage of CLL were also lower than those of WM and HCL, there were no significant differences between these groups. Many membrane markers have been used in B-CLPDs that can clearly distinguish between CLL, FL, MZL and HCL. However, CLL and MCL are both CD5-positive B-CLPDs, and it is sometimes difficult to distinguish CLL from MCL using morphology and immunophenotyping. Significant differences were observed in the MFI and expression percentage of CD35 $(\mathrm{P}<0.0001)$ between $C L L$ and MCL, suggesting that these 2 diseases could be differentiated by combining CD35 with effective markers (CD5, CD23, FMC7, CD22, CD79b, sIg). 


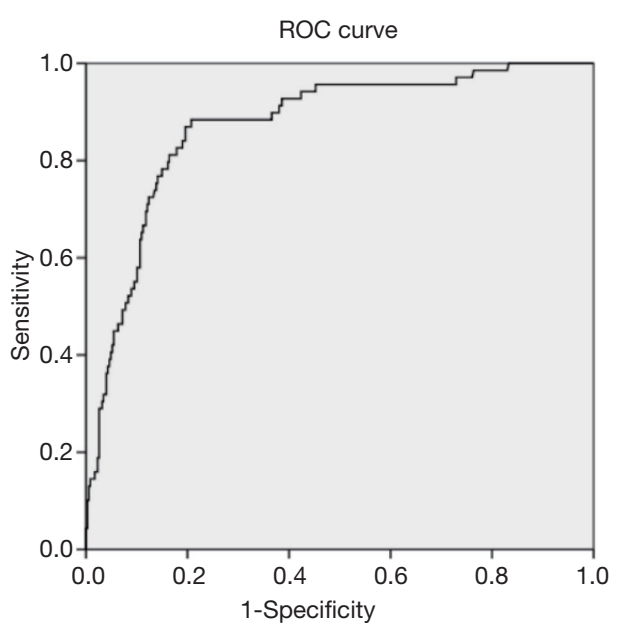

Figure 3 Receiver operating characteristic curve of CD35 expression to predict the diagnostic value of CD35 in differentiating CLL and MCL. A strong separation between these two subgroups in CD35 expression was reflected with an AUC of 0.873. AUC, area under the curve; CD, cluster of differentiation; CLL, chronic lymphocytic leukemia; MCL, mantle cell lymphoma.

Table $3 \chi^{2}$ analysis of CD35 expression in patients with CLL and MCL

\begin{tabular}{lcccc}
\hline CD35 & CLL $(\mathrm{n}=347)$ & $\mathrm{MCL}(\mathrm{n}=69)$ & Total $(\mathrm{n}=416)$ & $\mathrm{P}_{\text {value }}^{\dagger}$ \\
\hline Positive & 63 & 61 & 124 & $\mathrm{P}<0.001$ \\
Negative & 284 & 8 & 292 & \\
Total & 347 & 69 & 416 & \\
\hline
\end{tabular}

${ }^{\dagger}$ Significance tested is $\mathrm{P}<0.05$. CLL, chronic lymphocytic leukemia; MCL, mantle cell lymphoma.

Table 4 Patients classification according to Matutes score and the alternative proposed score

\begin{tabular}{cccccc}
\hline \multirow{2}{*}{ MS } & \multicolumn{2}{c}{ Matutes score } & & \multicolumn{2}{c}{ MS with CD35 added } \\
\cline { 2 - 3 } \cline { 5 - 6 } & CLL & MCL & & CLL & MCL \\
\hline 25 & 117 & 0 & 253 & 0 \\
4 & 165 & 0 & 54 & 4 \\
3 & 39 & 8 & 30 & 4 \\
2 & 23 & 27 & 8 & 31 \\
1 & 3 & 34 & 2 & 30 \\
0 & 0 & 0 & 0 & 0 \\
\hline
\end{tabular}

CLL, chronic Iymphocytic leukemia; MCL, mantle cell lymphoma.
The differential diagnostic value of CD35 for CLL and MCL

ROC curve analysis was used to determine the predictive value of CD35 for CLL and MCL. A strong separation between these two subgroups was evident according to an ROC plot with an AUC of 0.873 [95\% confidence interval (CI): $0.828-0.918$ ] for CD35 (Figure 3). CD35 <17\% had a sensitivity of $81.8 \%$ and a specificity of $88.4 \%$ for diagnosing CLL. CD35 expression was $<17 \%$ in $284 / 347$ of CLL cases, while it was $\geq 17 \%$ in 61 of 69 MCL cases. A statistically significant difference $(\mathrm{P}<0.001)$ in $\mathrm{CD} 35$ expression was observed between CLL and MCL patients (Table 3). CD35<17\% supported the diagnosis of CLL. In other words, CD35 was considered score 1 when the positive cell population was $<17 \%$. Of the 347 CLL cases in this study, 282 CLL cases had the typical CLL phenotype (Matutes score $\geq 4$ ), while 65 cases showed an atypical phenotype (Matutes score <4). The Matutes score of all patients with MCL was less than 4 . With the addition of CD35 to Matutes score, the Matutes score of 25 patients with atypical CLL changed from 3 to 4 , while 4 cases in MCL group had a modified MS of 4 (Table 4). When adding CD35 to CD5, CD23, CD22, FMC7 and sIgM, the sensitivity and accuracy of the MS improved from $81.3 \%$ to $88.5 \%$, and from $96.6 \%$ to $97.6 \%$, respectively, and a high specificity was maintained $(\mathrm{P}<0.05$; Table 5$)$.

\section{The C3 and C4 serum complement levels in B-CLPDs}

Several reports have shown the complement system in CLL patients to be deficient. Therefore, the C3 and C4 serum complement levels in 516 cases of B-CLPDs were evaluated: $15.0 \%(52 / 347)$ of CLL patients had a defective serum C3 level, while 21.9\% (37/169) of other B-CLPD patients had a defective serum $\mathrm{C} 3$ level at diagnosis. No significant difference was found between CLL and other $\mathrm{B}-\mathrm{CLPD}$ in $\mathrm{C} 3$ serum complement level deficiency $(\mathrm{P}=0.051$; Table 6). Similar results were also found in $\mathrm{C} 4$ component serum levels between CLL and other B-CLPDs (Table 7). Furthermore, 57 CLL cases had a defective serum C4 level while 38 other B-CLPD patients had a defective serum $\mathrm{C} 4$ level at diagnosis. In conclusion, a correlation was not found between $\mathrm{C} 3$ or $\mathrm{C} 4$ serum complement level deficiency and B-CLPD subtypes $(\mathrm{P}>0.05)$.

\section{Discussion}

Immunophenotyping by flow cytometry is a common 
Table 5 Sensitivity, specificity and accuracy of Matutes scoring systems in differential diagnosis between CLL and MCL

\begin{tabular}{lccc}
\hline Scoring system & Sensitivity \% (95\% Cl) & Specificity \% (95\% Cl) & CLL vs. MCL \% (95\% Cl) \\
\hline CD5, CD23, FMC7, Smlg, CD22 & $81.3(76.8-85.2)$ & $100.0(94.8-100.0)$ & $96.6(94.4-98.1)$ \\
CD5, CD23, FMC7, Smlg, CD22, CD35 & $88.5(84.6-91.6)$ & $94.2(85.8-98.4)$ & $97.6(95.6-98.8)$ \\
\hline
\end{tabular}

CLL, chronic lymphocytic leukemia; MCL, mantle cell lymphoma.

Table $6 \chi^{2}$ analysis of C3 component serum levels in patients with CLL and other B-CLPDs

\begin{tabular}{lcccc}
\hline C3 & CLL $(\mathrm{n}=347)$ & $\begin{array}{c}\text { Other B-CLPDs } \\
(\mathrm{n}=169)\end{array}$ & $\begin{array}{c}\text { Total } \\
(\mathrm{n}=516)\end{array}$ & $\mathrm{P}$ value $^{\dagger}$ \\
\hline Normal & 295 & 132 & 427 & $\mathrm{P}=0.051$ \\
Deficiency & 52 & 37 & 89 & \\
Total & 347 & 169 & 516 & \\
\hline
\end{tabular}

${ }^{\dagger}$ Significance tested is $\mathrm{P}<0.05$. CLL, chronic Iymphocytic leukemia; B-CLPD, B-chronic lymphoproliferative disorder.

Table $7 \chi^{2}$ analysis of $\mathrm{C} 4$ component serum levels in patients with CLL and other B-CLPDs

\begin{tabular}{lcccc}
\hline C4 & CLL $(n=347)$ & $\begin{array}{c}\text { Other B-CLPDs } \\
(n=169)\end{array}$ & Total $(n=516) P_{\text {value }}^{\dagger}$ \\
\hline Normal & 290 & 131 & 421 & $P=0.096$ \\
Deficiency & 57 & 38 & 95 & \\
Total & 347 & 169 & 516 & \\
\hline
\end{tabular}

${ }^{\dagger}$ Significance tested is $\mathrm{P}<0.05$. CLL, chronic lymphocytic leukemia; B-CLPD, B-chronic lymphoproliferative disorder.

and effective method for distinguishing CLL from other B-CLPDs. The diagnosis of CLL is definite when characteristic immunophenotypic features $\left(\mathrm{CD}^{+}, \mathrm{CD} 19^{+}\right.$, $\mathrm{CD} 23^{+}, \mathrm{CD} 22^{-/ \mathrm{dim}}, \mathrm{CD}_{79 b^{-/ d i m}}, \mathrm{FMC7}^{-}, \mathrm{sIg}^{\mathrm{dim}}$ and $\left.\mathrm{CD} 20^{\mathrm{dim}}\right)$ exist (4). However, making a differential diagnosis of CLL from other B-CLPDs is difficult in the absence of typical immunophenotypic features. The distinction between CLL, FL, MZL, and HCL can be made via membrane markers or different morphological characteristics. CLL and MCL are sometimes not easily distinguished by morphology and immunophenotyping. Some atypical phenotypes of CLL can be expressed as CD23 negativity, bright expression of CD20 or CD22, and FMC7 positivity $(20,21)$. Although CLL and MCL may share many similar morphologic and immunophenotyping features, the clinical evolution and therapeutic regimens of these two B-CLPD subtypes are divergent. Due to the difficulty in differentially diagnosing CLL and MCL, considerable research has been devoted to finding convenient and effective markers that might be used to distinguish CLL from MCL, such as CD18, CD54, and CD148 (22,23). Additional tests such as immunohistochemistry (IHC) for cyclin D1 or SOX11 and fluorescence in situ hybridization (FISH) analysis of $\mathrm{t}(11 ; 14)$ have been used to differentiate CLL from MCL.

CD35 is a complement receptor which can be expressed on B lymphocytes and play an important role in the regulation of humoral immunity $(14,24)$. Several studies have indicated that the expression of CD35 is significantly lower in patients with CLL. However, the sample capacity of these articles was small and did not compare the expression of CD35 in various B-CLPDs (18).

This study investigated the expression of CD35 on B leukemic cells of patients with CLL and other B-cell neoplasms by flow cytometry. The expression of CD35 was presented as an expression percentage, and MFI was calculated by analysis software. The results indicated that CD35 was expressed in B-CLPDs with significantly different expression levels. A high expression of CD35 was observed in MCL, FL, and MZL cases. CD35 expression of patients with WM and HCL was moderate, while CD35 had the lowest expression in CLL patients. The differences in CD35 expression between CLL and MCL, FL, MZL groups were statistically significant, while the differences between CLL and WM, HCL groups were not significant. The expression of CD35 was significantly different between CLL and other B-CLPDs which suggested that CD35 may be a useful marker in the differential diagnosis of CLL. Determining the predictive value of CD35 for CLL and MCL was the focus of this study. Subsequently, ROC curve analyses were conducted to assess CD35 expression for the diagnosis of CLL. The results showed that the sensitivity and specificity were up to $81.8 \%$ and $88.4 \%$ respectively at the cut-off value of $17 \%$. The classical Matutes score was effective in separating CLL from MCL when the Matutes score was high $(\geq 4)$, and 65 cases with atypical CLL overlapped with MCL at a Matutes score $\leq 3$. With 
the addition of CD35 to the Matutes score, 25 cases were reclassified into classical CLL and distinguished from MCL. Indeed, the alternative proposed score in this study achieved a high accuracy in CLL diagnosis.

It has been reported that CLL patients have a high incidence of an impaired complement system and low serum complement level. CLL patients are related to autoimmune features and poor prognosis $(25,26)$. Therefore, this study measured the $\mathrm{C} 3$ and $\mathrm{C} 4$ serum complement levels in 516 B-CLPD cases. It was found that $15.0 \%(52 / 347)$ and $16.4 \%$ (57/347) of CLL patients, respectively, had defective serum $\mathrm{C} 3$ and $\mathrm{C} 4$ levels, but there were no significant differences between CLL and other B-CLPDs in C3 and C4 serum complement level deficiency. Complement deficiencies in CLL patients are a critical clinical issue and have implications for monoclonal antibody (mAb) therapy. Complement deficiencies lead to the attenuated induction of complement-dependent cytotoxicity (CDC) and can reduce clinical efficacy of mAb therapy in CLL patients. Supplement of fresh-frozen plasma (FFP) in parallel with $m A b$ therapy can enhance the clinical efficacy of the $\mathrm{mAb}$ therapy via the restoration of complement levels to their normal state (27). Although many reports consider the supplement of FFP as beneficial to the treatment of CLL patients, supplying FFP has not been widely adopted in clinical trials and the treatment effect has not been as good as expected (28-30). CD35, a major cellular receptor for $\mathrm{C} 3 \mathrm{~b} / \mathrm{C} 4 \mathrm{~b}$, is essential for regulating complement system activation $(13,31)$. We thus speculate that ineffective FFP supplement therapy may be related to the lack of CD35 expression in CLL patients. In addition, CLL patients often have autoimmune system diseases, including common autoimmune cytopenias (AIC) and relatively less frequent autoimmune diseases, such as autoimmune hemolytic anemia (AIHA), immune thrombocytopenia (ITP), pure red cell aplasia (PRCA), Sjögren syndrome and AID-related arthritis (32). Reduced CD35 expression was also observed in patients with AIHA, Sjögren syndrome, paroxysmal nocturnal hemoglobinuria (PNH), and systemic lupus erythematosus (SLE) (33). Consequently, whether CLL patients being easily complicated with autoimmune diseases is associated with a deficiency of CD35 expression needs to be further studied.

At the time of diagnosis, patients with CLL should undergo risk stratification according to the CLL International Prognostic Index (CLL-IPI). Patients who have the low- and intermediate-risk CLL-IPI group should be monitored every 6-12 months, while those who have the high- and very high-risk CLL-IPI group should be provided with treatment in early period or monitored every 3-6 months. Previously, patients were mainly treated with standard chemoimmunotherapy regimens. In recent years, bruton's tyrosine kinase (BTK) inhibitors, phosphatidylinositol-3-kinase (PI3K) inhibitors, and B cell lymphoma-2 (BCL-2) inhibitors make a breakthrough in CLL treatment. Chimeric antigen receptor T-cell (CAR-T) therapy has also begun to show a certain effect. In our study, CD35 is only used as a diagnostic indicator. Whether there is a difference in treatment between CD $35^{+}$CLL and CD35 CLL needs to be further studied.

In conclusion, CD35 seems to be a useful additional marker for routine laboratory analysis in the diagnosis of ambiguous CLL cases. Furthermore, CD35 detection by flow cytometry analysis is convenient and inexpensive when peripheral blood and/or bone marrow samples are available. Based on our findings, we recommend adding CD35 to the B-CLPD flow cytometric routine panels in CLL diagnosis.

\section{Acknowledgments}

Funding: This work was supported by grants from the National Natural Science Foundation of China (No. 81370656).

\section{Footnote}

Reporting Checklist: The authors have completed the STARD reporting checklist. Available at https://dx.doi. org/10.21037/atm-21-3199

Data Sharing Statement: Available at https://dx.doi. org/10.21037/atm-21-3199

Conflicts of Interest: All authors have completed the ICMJE uniform disclosure form (available at https://dx.doi. org/10.21037/atm-21-3199). The authors have no conflicts of interest to declare.

Ethical Statement: The authors are accountable for all aspects of the work in ensuring that questions related to the accuracy or integrity of any part of the work are appropriately investigated and resolved. The study was conducted in accordance with the Declaration of Helsinki (as revised in 2013). The study was approved by the institutional ethics board of Jiangsu Province Hospital (No. 2021-SRFA-208) and individual consent for this 
retrospective analysis was waived.

Open Access Statement: This is an Open Access article distributed in accordance with the Creative Commons Attribution-NonCommercial-NoDerivs 4.0 International License (CC BY-NC-ND 4.0), which permits the noncommercial replication and distribution of the article with the strict proviso that no changes or edits are made and the original work is properly cited (including links to both the formal publication through the relevant DOI and the license). See: https://creativecommons.org/licenses/by-nc-nd/4.0/.

\section{References}

1. Hallek M, Shanafelt TD, Eichhorst B. Chronic lymphocytic leukaemia. Lancet 2018;391:1524-37.

2. Bosch F, Dalla-Favera R. Chronic lymphocytic leukaemia: from genetics to treatment. Nat Rev Clin Oncol 2019;16:684-701.

3. Scarfò L, Ferreri AJ, Ghia P. Chronic lymphocytic leukaemia. Crit Rev Oncol Hematol 2016;104:169-82.

4. Cao L, Liu Y, Lu JB, et al. A feedback circuit of miR-34a/ MDM4/p53 regulates apoptosis in chronic lymphocytic leukemia cells. Transl Cancer Res 2020;9:6143-53.

5. Salem DA, Stetler-Stevenson M. Clinical Flow-Cytometric Testing in Chronic Lymphocytic Leukemia. Methods Mol Biol 2019;2032:311-21.

6. Jalal SD. The contribution of CD200 to the diagnostic accuracy of Matutes score in the diagnosis of chronic lymphocytic leukemia in limited resources laboratories. PLoS One 2021;16:e0247491.

7. Hoffmann J, Rother M, Kaiser U, et al. Determination of CD43 and CD200 surface expression improves accuracy of B-cell lymphoma immunophenotyping. Cytometry B Clin Cytom 2020;98:476-82.

8. Ting YS, Smith S, Brown DA, et al. CD200 is a useful diagnostic marker for identifying atypical chronic lymphocytic leukemia by flow cytometry. Int J Lab Hematol 2018;40:533-9.

9. Matutes E, Wotherspoon A, Catovsky D. Differential diagnosis in chronic lymphocytic leukaemia. Best Pract Res Clin Haematol 2007;20:367-84.

10. Matutes E, Attygalle A, Wotherspoon A, et al. Diagnostic issues in chronic lymphocytic leukaemia (CLL). Best Pract Res Clin Haematol 2010;23:3-20.

11. Maddocks K. Update on mantle cell lymphoma. Blood 2018;132:1647-56.

12. Liu D, Niu ZX. The structure, genetic polymorphisms, expression and biological functions of complement receptor type 1 (CR1/CD35). Immunopharmacol Immunotoxicol 2009;31:524-35.

13. Rochowiak A, Niemir ZI. The structure and role of CR1 complement receptor in physiology. Pol Merkur Lekarski 2010;28:79-83.

14. Roozendaal R, Carroll MC. Complement receptors CD2 1 and CD35 in humoral immunity. Immunol Rev 2007;219:157-66.

15. Klickstein LB, Barbashov SF, Liu T, et al. Complement receptor type 1 (CR1, CD35) is a receptor for C1q. Immunity 1997;7:345-55.

16. Medof ME, Nussenzweig V. Control of the function of substrate-bound $\mathrm{C} 4 \mathrm{~b}-\mathrm{C} 3 \mathrm{~b}$ by the complement receptor Cr1. J Exp Med 1984;159:1669-85.

17. Smith BO, Mallin RL, Krych-Goldberg M, et al. Structure of the C3b binding site of CR1 (CD35), the immune adherence receptor. Cell 2002;108:769-80.

18. Marquart HV, Gronbaek K, Christensen BE, et al. Complement activation by malignant $\mathrm{B}$ cells from patients with chronic lymphocytic leukaemia (CLL). Clin Exp Immunol 1995;102:575-81.

19. Swerdlow SH, Campo E, Pileri SA, et al. The 2016 revision of the World Health Organization classification of lymphoid neoplasms. Blood 2016;127:2375-90.

20. Marti GE, Rawstron AC, Ghia P, et al. Diagnostic criteria for monoclonal B-cell lymphocytosis. Br J Haematol 2005;130:325-32.

21. Angelillo P, Capasso A, Ghia P, et al. Monoclonal B-cell lymphocytosis: Does the elderly patient need a specialistic approach? Eur J Intern Med 2018;58:2-6.

22. Deneys V, Michaux L, Leveugle P, et al. Atypical lymphocytic leukemia and mantle cell lymphoma immunologically very close: flow cytometric distinction by the use of CD20 and CD54 expression. Leukemia 2001;15:1458-65.

23. Fan L, Miao Y, Wu YJ, et al. Expression patterns of CD200 and CD148 in leukemic B-cell chronic lymphoproliferative disorders and their potential value in differential diagnosis. Leuk Lymphoma 2015;56:3329-35.

24. Molina H, Holers VM, Li B, et al. Markedly impaired humoral immune response in mice deficient in complement receptors 1 and 2. Proc Natl Acad Sci U S A 1996;93:3357-61.

25. Shvidel L, Vorst E, Berrebi A. Complement values in B chronic lymphocytic leukemia: prognostic significance and correlation with cell maturation stage. Leukemia 1998;12:635-6. 


\section{Page 10 of 10}

26. Miao Y, Zou YX, Gu DL, et al. SF3B1 mutation predicts unfavorable treatment-free survival in Chinese chronic lymphocytic leukemia patients. Ann Transl Med 2019;7:176.

27. Middleton O, Cosimo E, Dobbin E, et al. Complement deficiencies limit CD20 monoclonal antibody treatment efficacy in CLL. Leukemia 2015;29:107-14.

28. Klepfish A, Gilles L, Ioannis K, et al. Enhancing the action of rituximab in chronic lymphocytic leukemia by adding fresh frozen plasma: complement/rituximab interactions \& clinical results in refractory CLL. Ann N Y Acad Sci 2009; 1173:865-73.

29. Xu W, Miao KR, Zhu DX, et al. Enhancing the action of rituximab by adding fresh frozen plasma for the treatment of fludarabine refractory chronic lymphocytic leukemia. Int J Cancer 2011;128:2192-201.

30. Klepfish A, Rachmilewitz EA, Kotsianidis I, et al. Adding

Cite this article as: Shi Y, Liu L, Yang H, Chen X, Wang Y, Zhao S, Jin $\mathrm{H}, \mathrm{Wu}$ Y. The potential differential diagnosis value and clinical significance of CD35 expression in B-chronic lymphoproliferative disorders. Ann Transl Med 2021;9(14):1166. doi: 10.21037/atm-21-3199

\section{Shi et al. Differential diagnosis between CLL and other B-CLPDs}

fresh frozen plasma to rituximab for the treatment of patients with refractory advanced CLL. QJM 2008;101:737-40.

31. Carroll M. Role of complement receptors CD21/CD35 in B lymphocyte activation and survival. Curr Top Microbiol Immunol 1999;246:63-8; discussion 69.

32. Vitale C, Montalbano MC, Salvetti C, et al. Autoimmune Complications in Chronic Lymphocytic Leukemia in the Era of Targeted Drugs. Cancers (Basel) 2020;12(2).

33. Ross GD, Yount WJ, Walport MJ, et al. Disease-associated loss of erythrocyte complement receptors (CR1, C3b receptors) in patients with systemic lupus erythematosus and other diseases involving autoantibodies and/or complement activation. J Immunol 1985;135:2005-14.

(English Language Editors: D. Estela and J. Gray) 